\title{
A real time road marking detection system on large variability road images database
}

\begin{abstract}
For no less than two decades, the development of autonomous systems has led to the development of embedded applications permitting to enhance the driving comfort and limit the hazard level of dangerous zones. One of the first embedded system is a lane detection system, which was implemented using road marking detection algorithms with the aim to produce a system that is able to detect various shapes of road markings on the images that are captured under various imaging conditions. Generally, the road images were captured using a camera, which has been placed inside a vehicle at a fixed position. In this paper, a road markings detection system that tackles the problems of detecting road markings on the images captured under various weather and illumination conditions is proposed. The proposed system consists of inverse perspective transform method, which is used to convert an image into a birdôs-eye view image, an image normalization method, namely CLAHE that tackle various illumination conditions and Sobel edge detection method for identifying the road marker. We demonstrate the usefulness of the constructed algorithm by performing experiments on our Large Variability Road Images database (LVRI) that consists of 22,500 road images with the accuracy of $96.53 \%$.
\end{abstract}

Keyword: Road marks detection; Bird's eye view image; CLAHE; Sobel edge detection; LVRI database 